\title{
Theory of resonantly enhanced multiphoton processes in molecules
}

\author{
S. N. Dixit and V. McKoy \\ Arthur Amos Noyes Laboratory of Chemical Physics, ${ }^{a)}$ California Institute of Technology, Pasadena, California 91125
}

(Received 16 August 1984; accepted 5 October 1984)

In this paper we formulate a theory for the analysis of resonant enhanced multiphoton ionization processes in molecules. Our approach consists of viewing the $(n+m)$ photon ionization process from an isotropic initial state as $m$-photon ionization out of an oriented, excited state. The orientation in this resonant state, which is reached by $n$-photon excitation from the initial state, is nonisotropic, and is characteristic of this absorption process. The ionization simply probes this anisotropic population. The calculation of the REMPI process thus consists of determining the anisotropy created in the resonant state and then coupling this anisotropic population to ionization out of it. While the former is accomplished by the solution of appropriate density matrix equations, the latter is done by coupling these density matrix elements to angle-resolved ionization rates out of this state. An attractive feature of this approach is that the influence of saturation effects, and other interactions, such as collisions, on the photoelectron properties is easily understood and incorporated. General expressions are derived for photoelectron angular distributions. Based on these, several properties of the angular distributions that follow purely on symmetry considerations are discussed. One of the new features that emerge out of this work is the saturation induced anisotropy in REMPI. In this effect higher order contributions to the angular distributions appear since saturation influences different ionization channels differently thereby creating an additional anisotropy in the excited state.

\section{INTRODUCTION}

Multiphoton absorption and ionization processes are a powerful tool in the study of atomic and molecular systems. High power lasers commonly used in multiphoton processes not only increase the signal to noise ratio thereby making processes of very high order observable, but also provide extremely high energy and quantumstate selectivity. Such features are successfully being employed in areas such as excited state spectroscopy, ${ }^{1}$ single atom detection, ${ }^{2}$ laser isotope seperation, ${ }^{3}$ combustion diagnostics, ${ }^{4}$ studies of vibrational energy redistribution in complex molecules, ${ }^{5}$ and quantum-state-specific detection of scattered species in atom-molecule and moleculesurface scattering. ${ }^{6}$ The scope of multiphoton processes continues to grow as new applications both to basic and applied research are identified.

A resonant enhanced multiphoton ionization process (REMPI) is one in which the energy of an integral number of photons matches the energy difference between the initial state of the system and some intermediate state. The probability of ionization is enhanced due to the increase in the lifetime of the virtual intermediate state which now becomes the real excited state. This reduces the power levels required for their observation. While the field of resonant multiphoton processes in atomic systems

\footnotetext{
a) Contribution No. 7072 .
}

is quite mature with many of the observed effects understood qualitatively and some even quantitatively, ${ }^{7}$ the basic understanding of molecular multiphoton ionization processes is still in its infancy. Current experimental studies $^{8-20}$ of REMPI processes in molecules such as $\mathrm{H}_{2}$, $\mathrm{N}_{2}, \mathrm{NO}$, and $\mathrm{CO}$ have revealed several interesting features such as state selectivity in the residual ion, non-FranckCondon behavior, rotational state dependence of photoelectron angular distributions, and vibrational and rotational autoionization.

In this paper we present a formalism for the investigation of the REMPI process. Focusing on diatomic molecules, we establish a framework for carrying out REMPI calculations. As many of the REMPI experiments quoted above have a single intermediate resonant state, the formalism presented here is for a $(n+m)$ type REMPI process. Our approach is based upon viewing the $(n+m)$ REMPI of a molecule in its (isotropic) ground state as a $m$ photon ionization of the oriented, excited state. The orientation of this state, which is created by $n$ photon absorption from the ground state, carries in it the signature of the latter. Ionization then simply probes this anisotropic excited state. To illustrate the general features of the REMPI, without overburdening the algebra, we restrict ourselves to $(n+1)$-type REMPI. Such a process is also attractive both theoretically and experimentally as the probing of the excited state is "clean" and uninfluenced by accidental resonances in the ionization of the excited 
state that can occur in a $(n+m)$ type REMPI process. The generalization of this formalism to a $(n+m)$ type REMPI is relatively straightforward.

The calculation of the observable quantities in a ( $n$ $+1)$ REMPI can be separated into a part determining the orientation in the resonant intermediate state and into a part calculating photoionization out of this state. The details involved in this are described in Secs. II B and II C. Section II A describes the calculation of boundbound and bound-free matrix elements for a vibronic transition.

We believe that the present approach helps to make the physical interpretation of a $(n+1)$ REMPI process more transparent. Furthermore, inclusion of strong field effects and other interactions (e.g., collisions) altering the alignment of the resonant state is relatively straightforward. In light of experiments measuring the alignment of the excited state and its dependence on collisional perturbations, such an approach would be very useful in analyzing the experimental results so as to extract the excited state alignment. The present approach, however, does not include effects of autoionization. This, as well as the calculation of REMPI quantities for specific molecules will be discussed in future articles.

\section{THEORETICAL DEVELOPMENT}

\section{A. Wave functions and dipole matrix elements}

Let $H_{0}$ denote the molecular Hamiltonian in the absence of the radiation field and $V$ the interaction Hamiltonian between the molecule and the radiation field. Within the semiclassical approximation for the radiation and the electric dipole approximation for the interaction we can write $V$ as

$$
V=-\mu \cdot \mathbf{E}(t),
$$

where $\mathbf{E}(t)$ is the time-dependent electric field vector of the radiation field and $\mu$ denotes the dipole moment operator for the molecule. For the eigenfunctions of $H_{0}$ we use the Born-Oppenheimer approximation and write

$\left|\Psi_{\gamma J M K}\right\rangle=\sqrt{\frac{(2 J+1)}{8 \pi^{2}}} \psi_{\gamma}^{(e)}\left(\left\{\mathbf{r}_{i}^{\prime}\right\} ; R\right) \chi_{\nu}(R) D_{M K}\left(\eta_{K}(\hat{R})\right.$.

Here $\psi_{\gamma}^{(e)}$ is the electronic wave function that depends on the internuclear separation $R$ and $\chi_{\nu}(R)$ is the wave function for the vibrational state $\left.\nu . \mathscr{D}_{M K}^{(}\right)_{K}(\hat{R})$ denotes the symmetric top rotational wave functions with total angular momentum $J$, with the projection of this angular momentum along the $z$ axis of the space-fixed frame being $M$ and the projection along the $z$ axis in the body-fixed frame (which coincides with the internuclear axis) being $K$. ( $K=0$ for $\Sigma$ states, \pm 1 for $\Pi$ states, etc.). Throughout we shall follow the notation of Edmonds. ${ }^{21}$ Moreover, we will neglect the spin of the electron which will restrict our results to Hund's case (b). For singlet states, as is well known, Hund's cases (a) and (b) coincide and the results are applicable to case (a) as well. With $\psi_{\gamma}^{(e)}$ and $\chi_{v}$ normalized, the factor $\sqrt{(2 J+1) / 8 \pi^{2}}$ ensures the normal- ization of the total wave function. The primed coordinates refer to the molecule-fixed frame while the unprimed coordinates refer to the space fixed frame. $\gamma$ in Eq. (2) contains all other subscripts needed for an unambiguous designation of the state.

The transition matrix element between two bound states of the type given in Eq. (2) is defined as

$$
V_{21}=\left\langle\Psi_{\gamma_{2} J_{2} M_{2} K_{2}}|V| \Psi_{\gamma_{1} J_{1} M_{1} K_{1}}\right\rangle
$$

Writing

$$
\mathbf{E}(t)=E_{0}(t) e^{-i \omega t} \epsilon+\text { complex conjugate (c.c.), }
$$

where $\epsilon$ denotes the polarization vector and $E_{0}(t)$ the electric field envelope, and defining

$$
\boldsymbol{\mu}=e \sum_{s} \mathbf{r}_{s},
$$

where $\mathbf{r}_{s}$ is the position vector of the electron in the laboratory frame, we can write the interaction operator $V$ as

$$
V=-e\left\{E_{0}(t) e^{-i \omega t} \sum_{s} \mathbf{r}_{s} \cdot \boldsymbol{\epsilon}+\text { c.c. }\right\} .
$$

Let us first look at the $\Sigma_{s} \mathbf{r}_{s} \cdot \epsilon$ term. For polarized light this can be rewritten as

$$
D_{\mu 0}=\left(\frac{4}{3} \pi\right)^{1 / 2} \sum_{s} r_{s} Y_{\mu_{0}}\left(\hat{r}_{s}\right)
$$

where $\mu_{0}$ characterizes the polarization of the light: $\mu_{0}$ $=0$ for light linearly polarized along the $z$ axis in the laboratory frame while $\mu_{0}= \pm 1$ for circularly polarized light propagating along the $z$ axis, again in the laboratory frame. $Y_{1 \mu_{0}}\left(\hat{r}_{s}\right)$ can be transformed into the rotating molecular frame $\mathrm{e}^{21-23}$ using

$$
Y_{1 \mu 0}\left(\hat{r}_{s}\right)=\sum_{\mu}(-1)^{\mu-\mu_{0}} \mathcal{D}_{\mu \rho \mu}^{(1)}(\hat{R}) Y_{1 \mu}\left(\hat{r}_{s}^{\prime}\right)
$$

The matrix element of $D_{\mu_{0}}$ can then be written as

$$
\begin{aligned}
&\left\langle\psi_{\gamma_{2} J_{2} M_{2} K_{2}}\left|D_{\mu_{0}}\right| \psi_{\gamma_{1} J_{1} M_{1} K_{1}}\right\rangle \\
&=\left(\frac{4}{3} \pi\right)^{1 / 2} \sum_{\mu}(-1)^{\mu-\mu_{0}} \times\left\langle\psi_{\gamma_{2} J_{2} M_{2} K_{2}}\right| \\
& \times \sum_{s} r_{s} Y_{1 \mu}\left(\hat{r}_{s}^{\prime}\right) \mathcal{D}_{\mu_{0} \mu}^{(1)}(\hat{R})\left|\psi_{\gamma_{1} J_{1} M_{1} K_{1}}\right\rangle .
\end{aligned}
$$

With the wave functions of Eq. (2) and using Eq. (4.6.2) of Ref. 21 to carry out the integration over molecular orientation, this becomes

$$
\begin{aligned}
\left\langle\psi_{\gamma_{2} J_{2} M_{2} K_{2}}\left|D_{\mu_{0}}\right| \psi_{\gamma_{1} J_{1} M_{1} K_{1}}\right\rangle \\
=\left(\frac{4}{3} \pi\right)^{1 / 2} \sqrt{\left(2 J_{1}+1\right)\left(2 J_{2}+1\right)} \\
\quad \times \sum_{\mu}\left(\begin{array}{ccc}
J_{2} & 1 & J_{1} \\
-M_{2} & \mu_{0} & M_{1}
\end{array}\right)\left(\begin{array}{ccc}
J_{2} & 1 & J_{1} \\
-K_{2} & \mu & K_{1}
\end{array}\right) \\
\quad \times(-1)^{\mu-K_{2}+\mu_{0}+M_{2}} \overline{\frac{r_{1}^{(\mu)}}{(\mu)}}
\end{aligned}
$$


where

$$
\overline{r_{21}^{(4)}}=\int d R \chi_{\nu_{2}}^{*}(R) r_{21}^{(\mu)}(R) \chi_{\nu_{1}}(R),
$$

with $r_{21}^{(\mu)}(R)$ denoting the electronic transition matrix element

$r_{21}^{(\mu)}(R)=\left\langle\psi_{\gamma 2}^{(e)}\left(\left\{\mathbf{r}_{i}^{\prime}\right\} ; R\right)\left|\sum_{s} r_{s} Y_{1 \mu}\left(\hat{r}_{s}^{\prime}\right)\right| \psi_{\gamma 1}^{(e)}\left(\left\{\mathbf{r}_{i}^{\prime}\right\} ; R\right)\right\rangle$

at a fixed internuclear separation $R$.

In these $(n+1)$ REMPI studies, one needs, in addition to the bound-bound matrix elements of the interaction Hamiltonian $V$, the matrix elements of $V$ between the resonant intermediate state and the ionization continuum. The electronic wave function for the continuum electron needed in the calculation of this boundfree matrix element can be expanded in partial waves, ${ }^{22,23}$ as

$|\phi\rangle=\sum_{l m \lambda} i^{l} e^{-i \eta \prime}(-1)^{m-\lambda} Y_{m}^{*}(\hat{k}) \mathcal{D}_{m \lambda}^{(l)}(\hat{R}) \psi_{k l \lambda}\left(\mathbf{r}^{\prime} ; R\right)$,

$$
\begin{aligned}
\left\langle\psi_{\gamma_{f} J_{+} M_{+} K_{+}}\left|D_{\mu_{0}}\right| \psi_{\gamma_{i} J_{i} M_{i} K_{i}}\right\rangle= & \left(\frac{4}{3} \pi\right)^{1 / 2} \sum_{\substack { l m \lambda \\
\begin{subarray}{c}{\mu \\
j_{t} m_{t} k_{t}{ l m \lambda \\
\begin{subarray} { c } { \mu \\
j _ { t } m _ { t } k _ { t } } }\end{subarray}}(-i)^{l} e^{i \eta l} Y_{l m}(\hat{k})(-1)^{\mu-\mu_{0}+M_{+}-K_{+}+m_{t}-k_{t}}\left(2 j_{t}+1\right) \sqrt{\left(2 J_{i}+1\right)\left(2 J_{+}+1\right)} \\
& \times\left(\begin{array}{ccc}
J_{+} & J_{i} & j_{t} \\
-M_{+} & M_{i} & m_{t}
\end{array}\right)\left(\begin{array}{ccc}
l & 1 & j_{t} \\
-m & \mu_{0} & -m_{t}
\end{array}\right)\left(\begin{array}{ccc}
J_{+} & J_{i} & j_{t} \\
-K_{+} & K_{i} & k_{t}
\end{array}\right)\left(\begin{array}{ccc}
l & 1 & j_{t} \\
-\lambda & \mu & -k_{t}
\end{array}\right) \overline{r_{f i}^{(\mu)}}
\end{aligned}
$$

where $j_{t}$ corresponds to the angular momentum transferred to the ion with $m_{t}$ and $k_{t}$ denoting its projections along the laboratory-frame and molecular-frame $z$ axes, respectively. Again, as in Eqs. (10) and (11),

$$
\overline{r_{f i}^{(\mu)}}=\int \chi_{\nu_{+}}^{*}(R) \chi_{\nu_{i}}(R) d R \cdot r_{f i}^{(\mu)}(R),
$$

where

$r_{f i}^{(\mu)}(R)=\left\langle\psi_{\gamma f}^{(e)}\left(\left\{\mathbf{r}_{i}^{\prime}\right\} ; R\right)\left|\sum_{s} r_{s} Y_{1 \mu}\left(\hat{r}_{s}^{\prime}\right)\right| \psi_{\gamma i}^{(e)}\left(\left\{\mathbf{r}_{i}^{\prime}\right\} ; R\right)\right\rangle$

denotes the electronic transition moment for the $i \rightarrow f$ transition.

\section{B. Calculating the REMPI probability}

$\mathrm{A}(n+m)$-photon ionization from an isotropic initial state can be thought of as a $m$ photon ionization out of an oriented resonant intermediate state. The orientation is created as a result of absorption of $n$ photons from the initial state and carries within it the signature of the latter. The anisotropy or the alignment in the excited state is also sensitive to effects such as saturation and collisions. Such mechanisms will erase the $n$-photon absorption signature of the excited state thereby altering the ionization signal which probes the anisotropy of the excited state. A calculation of observable quantities such as the total ionization probability, the photoelectron energy and angular distributions in a REMPI process involves two steps: (a) calculation of the orientation in the resonant excited state. This involves the determination of the anisotropic population in the resonant excited state taking into account where $k$ denotes the momentum of the photoelectron and $\hat{k}$ its direction in the laboratory frame. $\operatorname{lm} \lambda$ denote, respectively, the angular momentum of the electron and its projections along the laboratory and molecular $z$ axes. The final state wave function can then be written as

$$
\begin{aligned}
\left|\psi_{\gamma f J_{+} M_{+} K_{+}}\right\rangle= & \sqrt{\frac{\left(2 J_{+}+1\right)}{8 \pi^{2}}} \chi_{\nu+}(R) D_{M_{+} K_{+}}^{\left.J_{+}\right)}(\hat{R}) \times \sum_{i m \lambda} i^{l} e^{-i \eta l} \\
& \times Y_{l m}^{*}(\hat{k})(-1)^{m-\lambda} \mathscr{D}_{m \lambda}^{(l)}(\hat{R}) \psi_{\gamma f}^{(e)}\left(\left\{\hat{r}_{i}^{\prime}\right\} ; R\right) .
\end{aligned}
$$

In the above equation $\nu_{+}, J_{+}, M_{+}$, and $K_{+}$denote, respectively, the ionic vibrational quantum number, its total angular momentum quantum number, the projection of $J_{+}$on the laboratory, and the molecular $z$ axes. $\psi_{\gamma f}^{e}\left(\left\{\hat{r}_{i}^{\prime}\right\} ; R\right)$ is the antisymmetrized electronic wave function including $\psi_{k l \lambda}\left(\hat{r}_{i}^{\prime} ; R\right)$ for the ionized electron and the $N-1$ electron wave function for the ion.

The matrix element of $D_{\mu_{0}}$ between a bound state $\left|\psi_{\gamma_{i} J_{i} M_{i} K_{i}}\right\rangle$ and the final continuum state $\left|\psi_{\gamma f J_{+} M_{+} K_{+}}\right\rangle$can then be written as the effects of saturation, ac Stark shifts, etc., and (b) photoionization out of this anisotropic excited state. The nonstatistical population distribution in the excited state calculated in (a) is coupled with the ionization rate out of this excited state to obtain photoionization probabilities, photoelectron energy, and angular distributions.

Let us consider a $(n+1)$ REMPI out of an initial state $\left|J_{0} M_{0}\right\rangle \equiv\left|\psi_{\gamma_{0} J_{0} M_{0} K_{0}}\right\rangle$. An isotropic population in this state implies that all the $M_{0}$ substates are equally populated. Let the resonant intermediate state reached after absorption of $n$ photons from $\left|J_{0} M_{0}\right\rangle$ be denoted by $\left|J_{i} M_{i}\right\rangle \equiv\left|\psi_{\gamma_{i} J_{i} M_{i} K_{i}}\right\rangle$. The $(n+1)$ th photon then ionizes this state $\left|J_{i} M_{i}\right\rangle$. For simplicity we shall restrict the intermediate state to have a specific $J_{i}$ value. Such an approximation is quite good for molecules with a large rotational spacing. For heavier molecules whose rotational spacing becomes comparable to or smaller than the laser bandwidth, more states with different $J_{i}$ allowed by the selection rules will have to be included in the calculation. The generalization of the present analysis to such cases involving more than one intermediate state can be done along the lines of Ref. 24.

For linearly polarized light $\mu_{0}=0$ and therefore $M_{i}$ $=M_{0}$. In the absence of collisions and other interactions that mix different magnetic substates, ionization out of each $\left|J_{0} M_{0}\right\rangle$ state forms an independent channel

$$
\left(\left|J_{0} M_{0}\right\rangle \stackrel{n \hbar \omega}{\rightarrow}\left|J_{i} M_{i}=M_{0}\right\rangle \stackrel{\hbar \omega}{\rightarrow}\right. \text { continuum). }
$$

Within the rotating wave approximation (RWA), the photoionization dynamics in each channel can be de- 
scribed by the following equations for the slowly varying density matrix elements ${ }^{24}$ :

$$
\begin{aligned}
& \frac{d}{d t} \rho_{00}=-\frac{1}{2} i\left(\Omega_{i i}^{(n)} \rho_{i 0}-\text { c.c. }\right), \\
& \frac{d}{d t} \rho_{i i}=-\Gamma_{i} \rho_{i i}+\frac{1}{2} i\left(\Omega_{\delta i}^{(n)} \rho_{i 0}-\text { c.c. }\right), \\
& {\left[\frac{d}{d t}-i \Delta+\frac{1}{2} \Gamma_{i 0}\right] \rho_{i 0}=\frac{1}{2} i \Omega_{i 0}^{(n)}\left(\rho_{i i}-\rho_{00}\right),}
\end{aligned}
$$

where

$$
\begin{aligned}
& \rho_{00}=\left\langle J_{0} M_{0}|\rho| J_{0} M_{0}\right\rangle \\
& \rho_{i i}=\left\langle J_{i} M_{i}|\rho| J_{i} M_{i}\right\rangle,
\end{aligned}
$$

and

$$
\rho_{\text {i0 }}=\left\langle J_{i} M_{i}|\rho| J_{0} M_{0}\right\rangle,
$$

and,

$$
\frac{1}{2} \Omega_{0 i}^{(n)}=\frac{1}{2} \Omega_{i}^{(n)}=\frac{E_{0}^{n}(t)}{\hbar} \sum_{\substack{\left|J_{1} M_{1}\right\rangle \\ \vdots \\\left|J_{n-1} M_{n-1}\right\rangle}} \frac{\mu_{i,(n-1)} \mu_{n-1, n-2} \cdots \mu_{10}}{\left(\hbar \omega_{0}+\hbar \omega-\hbar \omega_{1}\right)\left(\hbar \omega_{0}+2 \hbar \omega-\hbar \omega_{2}\right)\left(\hbar \omega_{0}+(n-1) \hbar \omega-\hbar \omega_{n-1}\right)}
$$

denotes the Rabi frequency for the $0 \rightarrow i$ transition. In Eq. (19) $\mu_{p q} \equiv\left\langle J_{p} M_{p}|\mu \cdot \epsilon| J_{q} M_{q}\right\rangle$, where $\mu=e \Sigma_{s} \boldsymbol{r}_{s}$ and $\left|J_{p} M_{p}\right\rangle$ stands for states of the molecule having energies $\hbar \omega_{p}$ that are reached from the ground state by absorption of $p$ photons. The dynamic detuning $\Delta$ in Eq. $(17 \mathrm{c})$ is defined as $\Delta=n \omega+\omega_{0}-\omega_{i}+S_{0}-S_{i}$, where $S_{0}$ and $S_{i}$ denote the ac Stark shifts of states $\left|J_{0} M_{0}\right\rangle$ and $\left|J_{i} M_{i}\right\rangle$, respectively. They are defined together with the corresponding ionization widths $\Gamma_{i}$ through

$$
\begin{aligned}
S_{i}-\frac{1}{2} i \Gamma_{i}= & \frac{1}{\hbar} \sum_{j} \frac{\left|\mu_{i j} E_{0}(t)\right|^{2}}{-\hbar \omega+\hbar \omega_{i}-\hbar \omega_{j}+i \epsilon} \\
& +\sum_{k} \frac{\left|\mu_{i k} E_{0}(t)\right|^{2}}{\hbar \omega+\hbar \omega_{i}-\hbar \omega_{k}+i \epsilon},
\end{aligned}
$$

where the summation over $j, k$ includes both bound and continuum states of the molecule and $\epsilon \rightarrow 0^{+}$. The ac Stark shift of the ground state is defined in a similar fashion.

At time $t=0$, the molecule is in its initial state which is assumed to be isotropic. Therefore $\rho_{00}(t=0)$ $=1 /\left(2 J_{0}+1\right)$ for all $M_{0}$. As different channels originating from different $\left|J_{0} M_{0}\right\rangle$ states do not mix, the time evolution of the probability in each channel is given by the solution of Eq. (17) with the above initial condition. It should be noted that although $\rho_{00}$ for various $M_{0}$ are equal at $t=0$, the populations $\rho_{00}$ and $\rho_{i i}$ will not be equal in different channels as $\Omega_{0 i}, S_{i}$, and $\Gamma_{i}$ are channel dependent. Thus the intermediate state is anisotropic although the initial state was isotropic at $t=0$. By the conservation of probability, the probability of ionization in a channel originating at $\left|J_{0} M_{0}\right\rangle$ is given by

$$
P_{M_{0}}(t)=\rho_{00}(t=0)-\rho_{00}(t)-\rho_{i i}(t),
$$

with $\rho_{00}(t)$ and $\rho_{i i}(t)$ being provided by Eq. (17) using molecular parameters for the corresponding channel. The total ionization probability (I.P.) is simply the sum total of the I.P. in each channel, i.e.,

$$
P=\sum_{M_{0}} P_{M_{0}} \text {. }
$$

The analytical solutions of Eq. (17) are well known and will not be reproduced here. If more than two levels participate in the dynamics of the REMPI process, Eq. (17) has to be suitably modified. Analytical solutions to such a larger system of equations cannot be found and one resorts to numerical solutions. Several interesting features of MPI have been demonstrated recently using numerical solutions. ${ }^{24}$

\section{REMPI photoelectron angular distributions}

Even though a knowledge of $\rho_{00}$ and $\rho_{i i}$ is sufficient to calculate the total ionization probability, it is not sufficient to calculate the detailed features in the ionization continuum. Features such as the photoelectron energy distribution and its angular distributions are averaged out in Eq. (17) as these equations are only sensitive to the total decay into the continuum and not to the details of this decay. Calculation of such details of the photoelectron signal then requires coupling of the population in the excited states obtained in the previous section to the photoionization out of this state. The ionization rate has to be summed only over the redundant variables while retaining the relevant details. To illustrate this, we shall focus on photoelectron angular distributions.

The rate of ejection of a photoelectron in the direction $(\theta, \phi)$ after ionization of a state $\left|J_{i} M_{i}\right\rangle$ is given by ${ }^{24}$

$$
\frac{d}{d t} P_{M_{i}}(\theta, \phi)=\Gamma_{M_{i} M_{i}}(\theta, \phi) \rho_{i i},
$$

where $\rho_{i i}$ is the population in state $\left|J_{i} M_{i}\right\rangle$ obtained from Eq. (17) and the differential ionization rate out of the state $\Gamma_{M_{i} M_{i}}(\theta, \phi)$ is defined as

$$
\begin{aligned}
\Gamma_{M_{i} M_{i}}(\theta, \phi)= & 2 \pi \alpha F(\hbar \omega) \\
& \times \sum\left|\left\langle\psi_{\gamma f J_{+} M_{+} K_{+}}\left|D_{\mu_{0}}\right| \psi_{\gamma_{i} J_{i} M_{i} K_{i}}\right\rangle\right|^{2} .
\end{aligned}
$$

In Eq. (24) $\alpha$ is the fine structure constant and the summation is over the redundant parameters in the final state. The spherical polar angles $\theta$ and $\phi$ are measured in a coordinate system whose $z$ axis coincides with the direction of light polarization for linearly polarized light and with the direction of propagation if the light is 
circularly polarized. $\Gamma_{M_{i} M_{i}}(\theta, \phi)$ is related to the more familiar differential cross section out of state $\left|J_{i} M_{i}\right\rangle$ through

$$
\Gamma_{M_{i} M}(\theta, \phi)=F \frac{d \sigma_{M_{i}}}{d \Omega_{k}},
$$

and is related to $\Gamma_{i}$ defined in Eq. (20) by

$$
\Gamma_{i}=\int \Gamma_{M_{i} M_{i}}(\theta, \phi) \sin \theta d \theta d \phi .
$$

The parameter $F$ in Eqs. (24) and (25a) denotes the photon flux (photons $/ \mathrm{cm}^{2} / \mathrm{s}$ ) and is related to the laser intensity $I\left(\mathrm{~W} / \mathrm{cm}^{2}\right)$ through $I=F(\hbar \omega)$.

Note that the summation in Eq. (24) runs over all the parameters of the continuum states which are not observed in a given experiment. For example, if the vibrational and/or rotational structure of the ion is not resolved, this implies summing over $\nu_{+}$and/or $J_{+}$. In recent experiments on REMPI in diatomic molecules, the vibrational structure of the residual ion is clearly resolved while the rotational structure is not. $J_{+}$can be detected by either measuring absorption or fluorescence from the ion. In the following analysis we will sum the right-hand side of Eq. (24) over $J_{+}$and $M_{+}$. Moreover, this is sufficient to describe the general features of the photoelectron angular distributions that follow purely on the basis of symmetry considerations.

It should be noted that the right-hand side of Eq. (23) is for ionization out of a single resonant intermediate state $\left|J_{i} M_{i}\right\rangle$. For situations where more than one inter- mediate state plays an important role in the ionization in a given channel, this equation has to be modified to

$$
\frac{d P_{M_{i}}(\theta, \phi)}{d t}=\sum_{\left|J_{i}^{\prime} M_{i}^{\prime}\right\rangle} \Gamma_{M_{i} M_{i}^{\prime}}(\theta, \phi) \rho_{i^{\prime} i},
$$

for each intermediate state. $\rho_{i^{i} i}$ must be calculated using a more complicated set of density matrix equations as described previously. ${ }^{24}$

Analogous to Eq. (24), $\Gamma_{M_{i} M^{\prime}}(\theta, \phi)$ is defined as ${ }^{25}$

$$
\begin{aligned}
\Gamma_{M i M^{\prime}}(\theta, \phi)= & 2 \pi \alpha F(\hbar \omega) \sum\left\langle\psi_{\gamma_{i} J_{i} M_{i} K_{i}}\left|D_{\mu_{0}}\right| \psi_{\gamma^{\prime} J+M+K_{+}}\right\rangle \\
& \times\left\langle\psi_{\gamma_{J} J_{+} M_{+} K_{+}}\left|D_{\mu_{0}}\right| \psi_{\gamma_{i}^{\prime} J_{i}^{\prime} M^{\prime} K_{i}^{\prime}}\right\rangle,
\end{aligned}
$$

where the summation is again over redundant final state variables.

The total probability $P(\theta, \phi)$ for electron ejection in the direction $\theta, \phi$ can then be calculated by adding up all the $P_{M}(\theta, \phi)$ for various $M_{i}$. Therefore,

$$
\frac{d P(\theta, \phi)}{d t}=\sum_{\substack{M_{i} \\ M_{i}^{\prime}}} \Gamma_{M_{i} M_{i}^{\prime}}(\theta, \phi) \rho_{i^{\prime} i}
$$

In a $n+m$ type REMPI $P(\theta, \phi)$ still obeys an equation similar to the above equation but $\Gamma_{M_{M} M_{i}^{\prime}}$ has to be modified to a differential $m$-photon ionization rate.

Using Eq. (14) for bound-free matrix elements of $D_{\mu 0}$ and Eq. (1.43) of Ref. 26 to combine a product of two spherical harmonics, the $\Gamma_{M_{i} M_{1}^{\prime}}(\theta)$ of Eq. (27) can be written as

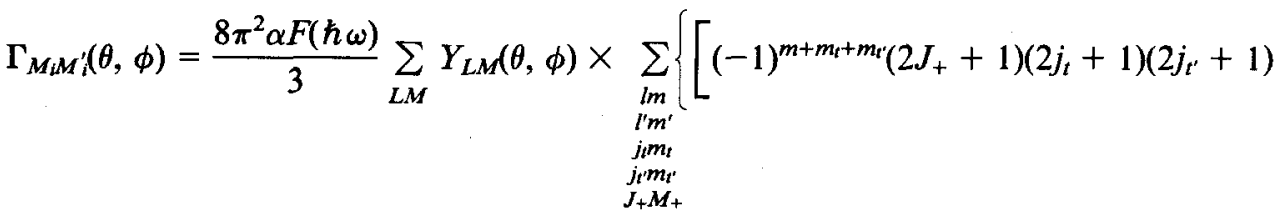

$$
\begin{aligned}
& \times \sqrt{\frac{\left(2 J_{i}+1\right)\left(2 J_{i}^{\prime}+1\right)(2 l+1)\left(2 l^{\prime}+1\right)(2 L+1)}{4 \pi}} \times\left(\begin{array}{lll}
l^{\prime} & l & L \\
0 & 0 & 0
\end{array}\right)\left(\begin{array}{ccc}
l^{\prime} & l & L \\
m^{\prime} & -m & M
\end{array}\right)\left(\begin{array}{ccc}
J_{+} & J_{i} & j_{t} \\
-M_{+} & M_{i} & m_{t}
\end{array}\right) \\
& \left.\times\left(\begin{array}{ccc}
l & 1 & j_{t} \\
-m & \mu_{0} & -m_{t}
\end{array}\right)\left(\begin{array}{ccc}
J_{+} & J_{i}^{\prime} & j_{t}^{\prime} \\
-M_{+} & M_{i}^{\prime} & m_{t}^{\prime}
\end{array}\right)\left(\begin{array}{ccc}
l^{\prime} & 1 & j_{i}^{\prime} \\
-m^{\prime} & \mu_{0} & -m_{t}^{\prime}
\end{array}\right)\right] \\
& \times\left[\sum_{\substack{\mu \mu^{\prime} \\
\lambda \lambda^{\prime}}}\left\{(-i)^{l} e^{i \eta \overline{r_{f}} r_{f_{i}}^{(\mu)}}\right\}\left\{(-1)^{\prime \prime} e^{i \eta \eta^{\prime} r_{f^{\prime}}^{(\mu)}}\right\}^{*}(-1)^{\mu+\mu^{\prime}-k_{t}-k_{t}^{\prime}}\left(\begin{array}{ccc}
J_{+} & J_{i} & j_{t} \\
-K_{+} & K_{i} & k_{t}
\end{array}\right)\left(\begin{array}{ccc}
l & 1 & j_{t} \\
-\lambda & \mu & -k_{t}
\end{array}\right)\right. \\
& \left.\left.\times\left(\begin{array}{ccc}
J_{+} & J_{i}^{\prime} & j_{t}^{\prime} \\
-K_{+} & K_{i}^{\prime} & k_{t}^{\prime}
\end{array}\right)\left(\begin{array}{ccc}
l^{\prime} & 1 & j_{t}^{\prime} \\
-\lambda^{\prime} & \mu^{\prime} & -k_{t}^{\prime}
\end{array}\right)\right]\right\} \equiv \sum_{L M} \gamma_{L M ; M_{i} M_{i}^{\prime}} Y_{L M}(\theta, \phi)
\end{aligned}
$$

Equation (28) together with Eq. (29) forms the main equation of our paper. In this form, Eq. (28) illustrates the basic nature of REMPI angular distributions: $(n+1)$ REMPI angular distributions can be thought of as single photon angular distributions out of an oriented excited state (all $\rho_{i i^{\prime}}$ are not equal). The degree of orientation induced in the excited state is contained in the values of $\rho_{i i^{\prime}}$. These $\rho_{i i^{\prime}}$ are sensitive not only to the $n$-photon absorption from the ground state but also to the effects of saturation, ac Stark shifts and to other mechanisms that influence the dynamics.
We now discuss some general features of the angular distributions that follow from Eqs. (28) and (29). However, we first note that the time evolution of $\rho_{i i}$ as described by Eq. (17) depends only on total loss of population to the continuum and not on the details of the loss into the continuum. Thus all the $\theta$ dependence of $P(\theta)$ comes from $\Gamma_{M_{i} M_{i}^{\prime}}(\theta)$ with $\rho_{i i^{\prime}}$ describing the population in the excited state. Ritchie et $a l^{27}$ recently have obtained angular distributions in three photon ionization of $\mathrm{H}_{2}$ by including a $\theta$ dependence of $\rho_{i i^{\prime}}$. The resulting "new 
features" are therefore erroneous and unphysical as has been pointed out by us elsewhere. ${ }^{28}$ Caution must therefore be used in generalizing expressions obtained in other contexts so as to describe angular distributions.

From Eqs. (28) and (29) it is clear that $P(\theta)$ itself can be expanded in spherical harmonics as

$$
P(\theta)=\sum_{L M}^{L_{\max }} \beta_{L M} Y_{L M}(\theta, \phi)
$$

Each $\beta_{L M}$ obeys an equation

$$
\frac{d \beta_{L M}}{d t}=\sum_{M_{i} M_{i}^{\prime}} \gamma_{L M ; M_{i} M_{i}^{\prime} \rho_{i^{\prime}}(t)}
$$

with a solution

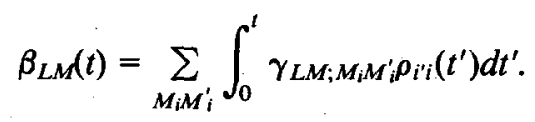

The sensitivity of $\beta_{L M}$ to saturation effects is clearly reflected through the dependence of $\rho_{i^{\prime} i}$ on these effects.

The coefficient of $Y_{L M}(\theta, \phi)$ in $\Gamma_{M_{i} M_{i}^{\prime}}(\theta)$ as written in Eq. (29) consists of two parts-a part that depends only on the projections of angular momenta on the $z$ axis in the laboratory frame and another part that depends on the projections along the molecule-frame $z$ axis. While the general symmetries are determined by the former, the actual details of the angular distributions are determined by the latter part which also contains the bound-free radial matrix elements. Although all possible values of $l$ are allowed in the partial wave expansion of the continuum wave function, only a finite number of these will contribute to $\Gamma_{M_{i} M_{i}^{\prime}}$ if the $M_{+}$of the ion is summed over. This in turn restricts the order of the spherical harmonics $L$ to a maximum value, say $L_{\max }^{(\Gamma)}$. An expression for $L_{\max }^{(\Gamma)}$ can be derived as follows. From Eq. (29) we have

$$
L_{\max }^{(\Gamma)}=\left(l+l^{\prime}\right)_{\max }=l_{\max }+l_{\max }^{\prime},
$$

where the second step follows from $l, l^{\prime} \geqslant 0$. Based on triangular relations imposed by the $3-j$ symbols Eq. (29) yields the following inequalities on $l, l^{\prime}$ if the $M_{+}$summation is not carried out:

$$
|| J_{+}-J_{i}|-1| \leqslant l \leqslant J_{+}+J_{i}+1
$$

and

$$
|| J_{+}-J_{i}^{\prime}|-1| \leqslant l^{\prime} \leqslant J_{+}+J_{i}^{\prime}+1 \text {. }
$$

These equations imply that as long as $J_{+}$is not restricted, i.e., rotational structure of the ion is not observed, $l, l^{\prime}$ are unrestricted making $L_{\max }^{(\Gamma)}$ unrestricted as well. In most experiments, however, even $J_{+}$is not resolved let alone $M_{+}$. The type of experiments that would resolve $M_{+}$and still measure $P(\theta)$ would consist of coincidence between the state of the ion (probed by the polarization of the fluorescence or by further absorption) and the corresponding photoelectron. Such experiments are difficult as the signal-to-noise ratio is greatly reduced. Therefore, we shall restrict ourselves to the case where the $M_{+}$state of the ion is not resolved. This allows us to sum Eq. (29) over $M_{+}$which severely restricts $L_{\max }^{(\Gamma)}$ as demonstrated below.

As $M_{+}$appears in only two $3-j$ symbols in Eq. (29), the summation over $M_{+}$can be rewritten, using Eq. (2.19) of Ref. 26, as

$$
\begin{aligned}
\sum_{M_{+}}\left(\begin{array}{ccc}
J_{+} & J_{i} & j_{t} \\
-M_{+} & M_{i} & m_{t}
\end{array}\right)\left(\begin{array}{ccc}
J_{+} & J_{i}^{\prime} & j_{t^{\prime}} \\
-M_{+} & M_{i}^{\prime} & m_{t^{\prime}}
\end{array}\right) \\
=\sum_{J_{M}}(-1)^{J_{+}+J-m_{t^{\prime}+M_{i}}(2 J+1)}\left\{\begin{array}{lll}
j_{t^{\prime}} & J_{i}^{\prime} & J_{+} \\
J_{i} & j_{t} & J
\end{array}\right\} \\
\quad \times\left(\begin{array}{ccc}
J_{i} & J_{i}^{\prime} & J \\
M_{i} & -M_{i}^{\prime} & M
\end{array}\right)\left(\begin{array}{ccc}
j_{t^{\prime}} & j_{t} & J \\
-m_{t^{\prime}} & m_{t} & -M
\end{array}\right) .
\end{aligned}
$$

While Eq. (29) implies

$$
l_{\max }+l_{\max }^{\prime}=\left(j_{t}\right)_{\max }+\left(j_{i}^{\prime}\right)_{\max }+2
$$

Eq. (37) above yields

$$
\left(j_{t}\right)_{\max }+\left(j_{t}^{\prime}\right)_{\max }=J_{\max }=\left(J_{i}\right)_{\max }+\left(J_{i}^{\prime}\right)_{\max } .
$$

Combining Eqs. (38a), (38b), and (35) we get

$$
L_{\max }^{(\Gamma)}=\left(J_{i}\right)_{\max }+\left(J_{i}^{\prime}\right)_{\max }+2 .
$$

If $J_{i}$ and $J_{i}^{\prime}$ take on single values only, then

$$
L_{\max }^{(\Gamma)}=J_{i}+J_{i}^{\prime}+2=\left(J_{i}+1\right)+\left(J_{i}^{\prime}+1\right) .
$$

This equation shows that the maximum allowed $L$ in $\Gamma_{i i}(\theta)$ depends only on $J_{i}, J_{i}^{\prime}$ with the 2 arising due to the angular momentum of the dipole photon. Physically this can be understood as follows. Since we are summing over $M_{+}$the anisotropy of the ionic state as it appears in Eq. (29) is averaged out. Thus all the anisotropy created in the final state $\left[J_{i}\right.$ due to the initial state and 1 due to the photon- $\left(J_{i}+1\right)$ maximum, similarly $\left.J_{i}^{\prime}+1\right]$ has to be exhibited by the electron. Hence the maximum allowed partial waves are $l_{\max }=J_{i}+1$ and $l_{\max }^{\prime}=J_{i}^{\prime}+1$ which implies $L_{\max }^{(\Gamma)}=J_{i}+J_{i}^{\prime}+2$.

Thus we have shown that if the $M_{+}$state of the ion is not resolved, then the maximum of $L$ in Eq. (29) is $J_{i}$ $+J_{i}^{\prime}+2$. Furthermore, if no $m$-mixing interactions are present, $M_{i}=M_{i}^{\prime}$ and this implies $M=0$. Therefore $P(\theta$, $\phi$ ) is independent of $\phi$ and consists only of Legendre polynomials. When the $(n+1)$ photon ionization takes place from a single state then $J_{i}=J_{i}^{\prime}$ and therefore

$$
L_{\max }^{(\Gamma)}=2 J_{i}+2 .
$$

For homonuclear linear molecules the allowed partial waves are either of $g$-symmetry (even $l, l^{\prime}$ ) or of $u$ symmetry (odd $l, l^{\prime}$ ). It follows from the properties of 3-j symbols that in each case only even $L$ 's are allowed in $\Gamma_{M_{i} M^{\prime}}(\theta)$. The same conclusion also holds true for heteronuclear molecules although the proof is tedious. Odd order spherical harmonics appear for molecules having fixed orientations in space $^{29}$ or for optically active molecules. ${ }^{30,31}$

Although Legendre polynomials of even order up to $L_{\max }^{(\Gamma)}$ appear in the expansions of $\Gamma_{M_{i} M^{\prime}}(\theta)$, the largest order $L_{\max }$ of Legendre polynomials in the averaged 
angular distribution [Eq. (30)] is determined by the orientation in the excited state ( $\rho_{i^{\prime} i}$ values). For example, if the state $\left|J_{i} M_{i}\right\rangle$ is isotropic, i.e.,

$$
\rho_{i^{\prime} i}=\frac{1}{\left(2 J_{i}+1\right)} \delta_{J_{i} J_{i}^{\prime} \delta_{M_{i} M_{i}^{\prime}}}
$$

as in the case of single photon ionization from an isotropic state with angular momentum $J_{i}$, one can show that $L_{\max }=2$. This agrees with earlier results ${ }^{22,23,32}$ and with Yang's theorem. ${ }^{33}$ The terms $\gamma_{L M ; M_{i} M_{i}^{\prime}}$ for $L>2$ add up to zero upon averaging over $M_{i}$ and $M_{i}^{\prime}$. Similarly, one can show, after tedious algebra, that if $\rho_{i i^{\prime}}$ carries in it the signature of single photon absorption from some state [weak field $1+1$ REMPI] $\left|J_{0} M_{0}\right\rangle$, i.e.,

$\rho_{i^{\prime} i} \propto \sum_{M_{0}} \frac{\left\langle J_{i}, M_{i},\left|D_{\mu_{0}}\right| J_{0} M_{0}\right\rangle\left\langle J_{0} M_{0}\left|D_{\mu_{0}}\right| J_{i} M_{i}\right\rangle}{\left(\hbar \omega_{0}+\hbar \omega-\hbar \omega_{i}\right)\left(\hbar \omega_{0}+\hbar \omega-\hbar \omega_{i^{\prime}}\right)}$,

where the state energies are independent of $M$ values, then $L_{\max }=4$. These relationships are nothing but restatements of Yang's theorem and its generalization for multiphoton ionization which states that in a $(n+1)$ photon ionization from an isotropic state, the maximum order of Legendre polynomials in the photoelectron angular distributions can be $2(n+1)$. Obviously $L_{\max }$ $\leqslant L_{\max }^{\left(I^{\prime}\right)}$ as $L_{\max }^{\left(I^{\prime}\right)}$ corresponds to highest anisotropy that can be measured in photoionization from state $\left|J_{i} M_{i}\right\rangle$. The above examples show that in weak field absorption $\rho_{i^{\prime} i}$ carries the specific signature of the $n$-photon absorption from the ground state which, when combined with ionization rate out of this excited state, yields $L_{\max }=2(n$ $+1)$. The actual shape of the angular distribution still depends on the values of the detailed bound-free matrix elements and hence on the molecular structure.

If the dependence of $\rho_{i^{\prime} i}$ on $M_{i}, M_{i}^{\prime}$ is different from the type shown in Eq. (43) then the angular distribution will be different. If $L_{\max }=L_{\max }^{(\Gamma)}$ the change will appear through variation of the coefficients $\beta_{L M}$ as has been demonstrated elsewhere in the case of atoms. ${ }^{24}$ If $L_{\max }<L_{\max }^{(\Gamma)}$ then additional terms in $P(\theta)\left(\beta_{L M} \neq 0\right.$ for $L$ $>L_{\max }$ ) may appear as the excited state anisotropy is of a different type.

We now discuss this appearance of higher order $\beta_{L M}$ in some detail. Saturation effects can alter the dependence of $\rho_{i^{\prime} i}$ on $M_{i} M_{i^{\prime}}$ from that predicted by lowest order perturbation theory thereby giving rise to higher order $\beta_{L M}$ 's. Such effects are expected to arise whenever light of high intensity is used to induce the REMPI process. For low intensities, the relative coherences between various $M_{i}$ channels leading to ionization are such that the $\beta_{L M}$ for $L>L_{\max }$ vanish. As the laser intensity is increased the $\rho_{i^{i} i}$ in each channel change differently as the leakage rates $\Gamma_{i}$ to the continuum are different. Moreover, the ac Stark shifts, which become significant at high intensities, are different for each channel as well since they explicitly depend on $M_{i}$ and $M_{0}$. Both these effects combine to destroy the specific relationships between $\rho_{i^{\prime} i}$ needed to cancel $\beta_{L M}$ for $L>L_{\max }$. Thus higher order contributions ( $\beta_{L M}$ for $L>L_{\max }$ ) appear in the angular distribution.
It is worth noting that higher order terms appear only when $L_{\max }<L_{\max }^{(\Gamma)}$. The initial state has a higher degree of anisotropy than can be probed by the REMPI. Saturation induced anisotropy then simply "exposes" this anisotropy. If the initial state, on the other hand, is purely isotropic $\left(J_{0}=0\right)$ and not in an averaged sense $\left(J_{0} \neq 0\right.$ and all $M_{0}$ 's equally populated) $L_{\max }=L_{\max }^{(\Gamma)}$ as the only anisotropy created is due to the absorption of photons. Saturation induced anisotropy in this case cannot expose any hidden (averaged-out) anisotropies as there are none. Similarly the largest $\beta_{L M}$ that could appear in $L=L_{\max }^{(\mathrm{r})}$ as this is the largest anisotropy that can be exposed.

Recent discussions of saturation effects ${ }^{24,34}$ on the angular distributions fall in the latter category since for alkali atoms the ground state is purely isotropic $(l=0)$. In this case $L_{\max }=L_{\max }^{(\mathrm{r})}$ and the effect of saturation is to alter $\beta_{L M}$ and not to introduce any higher order $\beta_{L M}$ 's. Atoms with non-s-type ground states or molecules with high angular momentum rotational states serve as good candidates to demonstrate this effect. What range of laser powers are needed to observe this effect of course depends on specific systems and the order of the process. Ab initio calculations are underway in our group to predict precise values of laser intensities and propose specific molecular systems to facilitate an experimental demonstration of this effect. The implication of such saturation induced anisotropics on other REMPI experiments is that one must be cautious in fitting experimental data to theoretical predictions based on lowest-order perturbation theory in extracting atomic/molecular parameters.

\section{SUMMARY AND FUTURE WORK}

In this paper we have laid down the ground work for a theoretical analysis of REMPI processes in linear molecules. The approach we have taken consists of viewing a $(n+1)$ type REMPI as single photon ionization of an excited oriented state, the orientation in which is created by a $n$-photon absorption from an isotropic initial state. Thus the orientation carries the signature of the REMPI process and the final ionization step simply probes it. We believe the present approach is very convenient and transparent for discussing intense field effects on photoelectron angular distributions. It is shown that additional terms may appear in the angular distributions under saturating conditions. Other approaches ${ }^{31}$ published in the literature are essentially low-order perturbation theory approaches and are, therefore, unable to discuss saturation effects.

Although several general features of angular distributions were deduced from symmetry considerations alone, the actual form of angular distributions depends on the values of the bound-free matrix elements $\vec{r}_{f i}^{(\mu)}$ and the phase shifts $\eta_{l}$. As such it will depend on the particular system under consideration. The effects of molecular structure will appear through the variations of $\vec{r}_{\text {if }}^{(\mu)}$ and $\eta_{l}$. Several experiments are underway presently that focus on a fundamental understanding of the REMPI process in simple diatomic molecules like $\mathrm{H}_{2}, \mathrm{CO}, \mathrm{NO}$, and $\mathrm{N}_{2}$. 
These experiments have revealed a number of interesting features such as the vibrational and rotational state dependence of angular distributions and non-Franck-Condon-like behavior in the vibrational distributions of the ions. In light of these results our work will focus on understanding of the REMPI in diatomic molecules. $A b$ initio calculations will be carried out to obtain the dynamical coefficients $\vec{r}_{f}^{(\mu)}$ and $\eta_{l}$ which, when combined with the theory presented in this paper, will enable us to compare the theoretical results with experiments.

\section{ACKNOWLEDGMENTS}

One of us (S.N.D.) acknowledges useful discussions with Peter Lambropoulos. This material is based upon research supported by the National Science Foundation under Grant No. CHE-8218166.

${ }^{1}$ See for example, J. Vergés et al., Phys. Rev. Lett. 53, 46 (1984); B. A. Garetz and C. Kittrell, ibid. 53, 156 (1984). See also the review by K. Kimura, in Adv. Chem. Phys. (to be published).

${ }^{2}$ G. W. Hurst, M. G. Payne, S. D. Kramer, and J. P. Young, Rev. Mod. Phys. 51, 767 (1979).

${ }^{3}$ V. N. Bagratashvili, V. S. Doljikov, V. S. Letokov, and E. A. Ryabov, Appl. Phys. 20, 231 (1979); I. P. Herman and J. B. Marling, Chem. Phys. Lett. 64, 75 (1979); J. Chem. Phys. 72, 516 (1980).

${ }^{4}$ See for example, D. Crosley, Bull. Am. Phys. Soc. 27, 862 (1982); J. E. M. Goldsmith, ibid. 27, 862 (1982).

${ }^{5}$ See for example, A. H. Zewail, Faraday Discuss. Chem. Soc. 75, 315 (1983), and references therein.

${ }^{6}$ E. E. Marinero, C. T. Rettner, and R. N. Zare, Phys. Rev. Lett. 48, 1323 (1982).

${ }^{7}$ See, for example, Multiphoton Ionization of Atoms, edited by S. L. Chin and P. Lambropoulos (Academic, New York, 1984); A. T. Georges and P. Lambropoulos, Adv. Electron. Electron Phys. 54, 191 (1980); P. Lambropoulos, Adv. At. Mol. Phys. 12, 87 (1976).

8 J. C. Miller and R. N. Compton, J. Chem. Phys. 75, 22 (1981).

9 J. Kimman, P. Kruit, and M. J. Van der Wiel, Chem. Phys. Lett. 88, 576 (1982).
${ }^{10}$ M. G. White, M. Seaver, W. A. Chupka, and S. D. Colson, Phys. Rev. Lett. 49, 28 (1982).

"J. C. Miller and R. N. Compton, Chem. Phys. Lett. 93, 453 (1982).

${ }^{12}$ S. T. Pratt, E. D. Poliakoff, P. M. Dehmer, and J. L. Dehmer, J. Chem. Phys. 78, 65 (1983).

${ }^{13}$ S. T. Pratt, P. M. Dehmer, and J. L. Dehmer, J. Chem. Phys. 78, 4315 (1983).

${ }^{14}$ S. T. Pratt, P. M. Dehmer, and J. L. Dehmer, J. Chem. Phys. 79, 3234 (1983).

${ }^{15}$ Y. Achiba, K. Sato, K. Schotabake, and K. Kimura, J. Chem. Phys. 78, 5474 (1983).

${ }^{16}$ M. G. White, W. A. Chupka, M. Seaver, A. Woodward, and S. D. Colson, J. Chem. Phys. 80, 678 (1984).

${ }^{17}$ S. T. Pratt, P. M. Dehmer, and J. L. Dehmer, J. Chem. Phys. 80, 1706 (1984).

${ }^{18}$ S. L. Anderson, G. D. Kubiak, and R. N. Zare, Chem. Phys. Lett. 105, 22 (1984)

${ }^{19}$ S. T. Pratt, P. M. Dehmer, and J. L. Dehmer, Chem. Phys. Lett. 105, 28 (1984).

${ }^{20}$ S. T. Pratt, P. M. Dehmer, and J. L. Dehmer, J. Chem. Phys. 81, 3444 (1984).

${ }^{21}$ A. R. Edmonds, Angular Momentum in Quantum Mechanics, 2nd ed., 3rd printing (Princeton University, Princeton, 1974).

${ }^{22}$ Y. Itakawa, Chem. Phys. 28, 461 (1978).

${ }^{23}$ A. D. Buckingham, B. J. Orr, and J. M. Sichel, Philos. Trans. R. Soc. London Ser. A 268, 147 (1970).

${ }^{24}$ S. N. Dixit and P. Lambropoulos, Phys. Rev. A 27, 861 (1983).

${ }^{25}$ For states with $K_{i}, K_{i}^{\prime} \neq 0, \Lambda$ degeneracy combined with parity considerations give rise to additional terms in the expressions for $\Gamma_{M M t}(\theta, \phi)$ [Eq. (24)], and for $\Gamma_{M M i}(\theta, \phi)$ [Eq. (27)] as has been discussed in Ref. 31. Although the general features discussed here depend only on angular momentum projections along the space fixed $z$ axis ( $M$ values), these extra terms will have to be carefully incorporated in an actual calculation of angular distributions for specific systems.

${ }^{26} \mathrm{M}$. Rotenberg, R. Bivins, N. Metropolis, and J. K. Wootens, Jr., $3 j$ and $6 j$ Symbols (Technology, Cambridge, MA, 1959).

${ }^{27}$ B. Ritchie, E. J. McGuire, J. M. Peek, and C. W. Band, J. Chem. Phys. 77, 877 (1982).

${ }^{28}$ S. N. Dixit and V. McKoy, J. Chem. Phys. 80, 5867 (1984).

${ }^{29}$ D. Dill, J. Chem. Phys. 65, 1130 (1976).

${ }^{30}$ B. Ritchie, Phys. Rev. A 13, 141 (1976); 14, 1396 (1976).

${ }^{31}$ J. C. Hansen and R. S. Berry, J. Chem. Phys. 80, 4078 (1984).

32 J. C. Tully, R. S. Berry, and B. J. Dalton, Phys. Rev. 176, 95 (1968).

${ }^{33}$ C. W. Yang, Phys. Rev. 74, 764 (1948).

${ }^{34}$ W. Ohnesorge, F. Diedrich, G. Leuchs, D. S. Elliott, and H. Walther, Phys. Rev. A 29, 1181 (1974). 